\title{
Percutaneous Dilational Tracheostomy in the Emergent Setting
}

\author{
Andrew McCague*, David T. Wong
}

Arrowhead Regional Medical Center, Colton, USA.

Email: *mccaguea@gmail.com

Received October $15^{\text {th }}$, 2012; revised November $15^{\text {th }}, 2012$; accepted November $22^{\text {nd }}, 2012$

\begin{abstract}
Objective: Since its inception, the use of the percutaneous dilational tracheostomy (PDT) has been contraindicated in the setting of an emergent airway. Emerging in the literature are several cases of successful emergent PDTs. Here we present our experience with the use of PDT in managing emergent airways. Study Design: All patients who underwent emergent PDT, using the Ciaglia Blue Rhino Introducer Set (Cook Critical Care, Bloomington, IN), in an academic county hospital setting between February 2010 and May 2012 were included in the study. Electronic medical records were reviewed for demographic and procedural data. Results: Twelve patients were included in the study with ages ranging from 20 to 87 (mean 57) years old. The most common reason for emergent airway was trauma (7 patients), followed by obstructing neck mass (2 patients), septic shock (2 patients), and angioedema (1 patient). Seven PDTs were performed in the OR, four at bedside and one in the ER. Three of the 12 patients had emergent cricothyroidotomies in place that malfunctioned, requiring emergent conversion. No patients suffered from short term complications. One patient developed a neck abscess at the site of the PDT one month post operatively, two patients had accidental decanulation post operatively, and both were replaced without complication. Conclusions: PDT can be used in the emergent setting in the hands of trained practitioners with minimal complications. A larger, prospective trial is needed to make conclusions regarding patient care.
\end{abstract}

Keywords: Percutaneous Dilational Tracheostomy; Emergent Airway; Tracheostomy

\section{Introduction}

The use of percutaneous dilational tracheostomy (PDT) has continuously increased since its original description by Ciagla in 1985. Since its inception, a need for an emergent airway has been considered an absolute contraindication for the use of PDT. As case reports emerge, a growing body of evidence is showing that PDT may be an option in specific emergent conditions where an emergent airway is necessary.

Most published articles consider cervical injury, pediatric age $<8$ years old, gross distortion of the neck anatomy, unidentifiable landmarks, visible large blood vessels in the operative field, and need for emergent airways as absolute contraindications, whereas obesity with a short neck, coagulopathy, need for positive end-expiratory pressure of $>20 \mathrm{~cm}$ of water, and evidence of infection in the soft tissues of the neck at the prospective surgical site are relative contraindications [2].

With difficult intubations occurring in approximately $2 \%-4 \%$ of all intubation attempts, the clinician may expect emergent surgical airways to periodically be re-

*Corresponding author. quired [2]. Unfortunately, most clinicians find themselves performing these procedures for the first time in an emergency, where failure rates and complication rates are already high [2]. The opportunity to perfect the technique of PDT in an elective setting improves performance of this technique during an emergent setting.

Here we present our experience with the use of PDT in managing emergent airways.

\section{Methods}

All patients who underwent emergent PDT, using the Ciaglia Blue Rhino Introducer Set (Cook Critical Care, Bloomington, IN), in an academic county hospital setting between February 2010 and May 2012 were included in the study. Electronic medical records were reviewed for demographic and procedural data. Emergent PDTs were defined as PDTs performed in the setting of impending airway loss. Short term complications were defined as bleeding, malposition, pneumothorax, tracheal ring rupture, conversion to open and procedural hypoxia. Long term complications included bleeding, infection, accidental decanulation, granuloma formation, tracheal stenosis 
and dysphagia.

All PDTs were performed with attending physician at bedside, most by a surgical resident. The Ciaglia Blue Rhino Single Dilator Introducer Kit ${ }^{\mathrm{TM}}$ (Cook Medical Inc., Bloomington, IN.) was used for all PDTs. All PDTs were performed without bronchoscopic guidance with local anesthetic and minimal or no sedation. When available, positioning was confirmed with bronchoscopy.

Follow-up was determined from the most recent visit as recorded in the electronic medical records. All data was recorded and stored in an Excel (Microsoft, Redmond Wa.) spreadsheet for descriptive analysis.

\section{Results}

Results are presented in Table 1 below. Twelve patients were included in the study with ages from 20 to 87 (mean 57) years old, length of stay 5 to 114 (mean 30) days and body mass index from 23.9 to 47 (mean 31 ) $\mathrm{kg} / \mathrm{m}^{2}$. The most common reason for emergent airway was trauma (7 patients), followed by obstructing neck mass (2 patients), septic shock (2 patients), and angioedema (1 patient). Seven PDTs were performed in the OR, four at bedside and one in the ER. Three of the 12 patients had emergent cricothyroidotomies in place that malfunctioned, requiring emergent bedside conversion.

No patients suffered from short term complications. No patient expired or suffered anoxic brain injury as a result of the procedure. One patient expired six days after the PDT was performed due to a pulmonary embolism. Six patients were discharged home, four sent to rehab, one transferred to another hospital and one expired. Long term complications occurred in 3 of the 12 patients. One patient developed a neck abscess at the site of the PDT one month post operatively, two patients had accidental decanulation post operatively, and both were replaced without complication. Follow-up ranged from 6 to 658 (mean 132) days.

\section{Discussion}

The use of PDT in the emergent setting has been revered as an absolute contraindication since its inception. As the use of PDTs has grown in recent years, a growing body of literature has shown the successful use of PDTs in the emergent setting. Though PDTs will not replace the emergent cricothyroidotomy, in specific clinical scenarios we have shown that the PDT can be safely used in an emergent setting avoiding the need for a future conversion procedure.

In the above study, twelve patients underwent PDT in an emergent setting without any short term complications. Three patients suffered from long term complications though these are unlikely related to the emergent nature of the procedure. One patient had a neck abscess and two patients had accidental decanulation. These complications could equally have occurred if the procedure was elective. One patient expired during the study due to a

Table 1. Descriptive data.

\begin{tabular}{|c|c|c|c|c|c|c|c|c|c|c|}
\hline Case & Age & Sex & Diagnosis & Indication & Location & Los & Follow up & Discharge & Confirmation bronchoscopy & Complication \\
\hline 1 & 78 & M & Cancer & $\begin{array}{l}\text { Difficult } \\
\text { intubation }\end{array}$ & ER & 5 & 193 & Home & No & Abscess \\
\hline 2 & 45 & $\mathrm{~F}$ & Angioedema & $\begin{array}{l}\text { Difficult } \\
\text { intubation }\end{array}$ & OR & 5 & 97 & Home & Yes & Decanulation \\
\hline 3 & 50 & M & Trauma & $\begin{array}{l}\text { Obstructed } \\
\text { Cricothyroidotomy }\end{array}$ & Bedside & 24 & 659 & Rehab & Yes & None \\
\hline 4 & 20 & M & Trauma & $\begin{array}{l}\text { Obstructed } \\
\text { cricothyroidotomy }\end{array}$ & Bedside & 9 & 31 & Home & No & Decanulation \\
\hline 5 & 80 & M & Trauma & $\begin{array}{l}\text { C-spine deformity, } \\
\text { difficult intubation }\end{array}$ & OR & 15 & 28 & Rehab & Yes & None \\
\hline 6 & 60 & M & Trauma & $\begin{array}{l}\text { Tracheal laceration } \\
\text { extending over ET tube }\end{array}$ & OR & 8 & 28 & Home & Yes & None \\
\hline 7 & 87 & $\mathrm{~F}$ & Trauma & $\begin{array}{l}\text { Difficult airway } \\
\text { impingment }\end{array}$ & OR & 19 & 245 & Hospital Transfer & Yes & None \\
\hline 8 & 61 & M & Sepsis & $\begin{array}{l}\text { Edematous airway, } \\
\text { failed intubation }\end{array}$ & Bedside & 90 & 80 & Home & Yes & None \\
\hline 9 & 49 & M & Sepsis & Obesity & Bedside & 114 & 158 & Rehab & Yes & None \\
\hline 10 & 27 & $\mathbf{M}$ & Trauma & GSW to Neck & OR & 6 & 6 & Expired & Yes & None \\
\hline 11 & 62 & M & Cancer & $\begin{array}{l}\text { Larygneal Cancer, } \\
\text { Difficult intubation }\end{array}$ & OR & 34 & 34 & Home & Yes & None \\
\hline 12 & 66 & $\mathrm{~F}$ & Trauma & $\begin{array}{l}\text { C-spine fx, } \\
\text { difficult airway }\end{array}$ & OR & 31 & 31 & Rehab & Yes & None \\
\hline
\end{tabular}


pulmonary embolism, unrelated to the use of PDT. For difficult to intubate patients with impending airway loss PDT is a possible alternative to cricothyroidotomy when performed in the hands of experienced practitioners.

In 2003, Ault et al. reported the largest series to date of successful emergent PDT which included nine patients with compromised airways. In their paper all nine patients were successfully intubated with a PDT technique either in the Emergency Room or one of the Intensive Care Units. They conclude that in specific clinical situations the PDT technique may play a role in management of emergent surgical airways [2].

Because of the emergent nature of the PDTs performed in our study, bronchoscopy was not used to guide placement. When available we did, however, use bronchoscopy to confirm placement. In the emergent situation, neither time nor the presence of an existing endotracheal tube permits the use of broncoscopy [2]. Bronchoscopic guidance is a valuable but not essential adjunct to performance of PDT and should not preclude use of this procedure in the emergent setting [2]. In 2011, Jackson et al. reported on 168 PDTs performed without bronchoscopy compared with 78 performed with bronchoscopy [3]. They found that there was not a difference in complication rates between the two groups thus confirming the practice of PDTs without bronchoscopy [3].

Though cricothyroidotomy is still the standard of care for an emergent surgical airway; PDT has several advantages. The major advantage of PDTs is the ability to gain and maintain competence in an elective and controlled setting [2]. This experience is invaluable as comfort and familiarity with any procedure improves success and reduces complications. Indications for performing a cricothyroidotomy (surgical or needle) are very limited. It is therefore extremely difficult to gain experience and become confident in performing this procedure [4]. The PDT also has the possibility of becoming a definitive airway for the patient, there is no need to convert to a formal tracheostomy as is the case with the cricothryroidotomy. PDT has the advantage over an open surgical technique as it is faster, can be performed under local anesthesia at the bedside with or without prior airway access, and can be performed within three minutes [4].

When comparing PDT to open tracheostomy, not only is PDT a faster procedure but it has also been reported to have lower complication rates. Moe et al. demonstrated that PDTs have lower rates of pneumothorax, major hemorrhage and laryngeal granulation when compared to open tracheostomy [2]. They also reported an 8 minute shorter procedure with PDTs as compared with open tracheostomy [2]. Graha et al. also reported similar advantages with the use of PDT. They found no significant difference in major or minor complications when comparing PDT to open tracheostomy [2].

Our experience with PDT has shown that difficult airways can be managed with this technique safely. In our series of twelve patients, all attempted emergent PDTs were performed successfully without loss of airway or need to convert to a conventional tracheostomy. Though additional trials are necessary to provide conclusive evidence, we suggest that the use of PDTs may have a place in the management of difficult and emergent airways.

\section{Conclusion}

Here we present twelve patients all who have undergone PDT in an emergent setting. None of these patients suffered short term complications. Long term complications seen in these patients were not related to the emergent nature of the procedure. PDT can be used in the emergent setting in the hands of trained practitioners with minimal complications. Our study shows a lower complication rate compared to previously published reports. A larger, prospective trial is needed to make conclusions regarding patient care.

\section{REFERENCES}

[1] A. McCague, H. Aljanabi and D. T. Wong, "Safety Analysis of Percutaneous Dilational Tracheostomies with Bronchoscopy in the Obese Patient," Laryngoscope, Vol. 122, No. 5, 2012, pp. 1031-1034. doi:10.1002/lary.22505

[2] M. J. Ault, B. Ault and P. Ng, "Percutaneous Dilational Tracheostomy for Emergent Airway Access,” Journal of Intensive Care Medicine, Vol. 18, No. 4, 2003, pp. 222226. doi:10.1177/0885066603254108

[3] L. S. Jackson, J. W. Davis, K. L. Kaups, L. P. Sue, M. M. Wolfe, J. F. Bilello and D. Lemaster, "Percutaneous Tracheostomy: To Bronch or Not to Bronch-That Is the Question,” Journal of Trauma, Vol. 71, No. 6, 2011, pp. 1533-1536. doi:10.1097/TA.0b013e31823ba29e

[4] N. Jonas, W. Mulwafu and J. Joubert, "Emergent Percutaneous Tracheostomy," South African Journal of Science, Vol. 45, No. 3, 2007, pp. 105-106. 